\title{
Cognitive computer graphics for information interpretation in real time intelligence systems
}

\author{
Yu.I. Nechaev, A.B. Degtyarev, A.V. Boukhanovsky \\ Institute for High Performance Computing and Data Bases \\ 120 Fontanka, St. Petersburg, 198005, Russia \\ E-mail: \{int, deg,avb\}@fn.csa.ru
}

\begin{abstract}
Application of cognitive computer graphics (CCG) in problems of information analysis and interpretation in real-time intelligence systems (IS) is considered. Using of cognitive structures at investigation of complex dynamic processes determining interaction of floating dynamic object (DO) with environment in extreme situations is in the focus of attention.
\end{abstract}

\section{Introduction and problem to solve}

CCG is connected with development of representation of symbolical and graphic objects by which geometrical thinking operates. Scientific basis of CCG is founded by A. Zenkin [16,17] and D. Pospelov. These ideas have developed in papers $[1,2,5,6,7,10,12,14,15]$. Despite of several publications devoted to CCG problem, practical applications of obtained theoretical solutions in onboard real-time IS are not numerous, e.g. $[6,7,14]$, probably, due to complexity of cognitive modeling of DO interaction with environment.

Let us present knowledge process as IS functioning [5,10,15]. Let us consider dynamically varied subject domain. Theoretical bases of cognitive structures formation in this area we shall develop using system of associated concepts determined on conceptual carcass. System of axioms as a finite set of statements is considered also. Due to large volume of processable information model of cognitive structure is preferable than isomorphic model. Basic principle of such model functioning is based on elimination of conflicts between observed data (results of dynamic measurements) and abstract theoretical knowledge [7,12].

One of the possible approaches to such modeling is using of integrated systems of Image Understanding (IUE - Image Understanding Environment) [3,11], based on definition of "standard" image objects and reflections between them. In the paper figurative representation of visual information as base component of intelligent block for complex onboard IS is considered. Figurative analysis as distinct from visual analysis does not use only visual human perception. It uses also special expert systems of figurative information arrays processing. These arrays represent ordered vector-matrix information containing characteristics of considered DO behaviour. 
Image models as cognitive structures are formed on the basis of such information [14].

Cognitive paradigm promotes to effective dialogue between operator and computer. It is important approach to knowledge formalisation in various IS stimulating finding of original technical decisions and generation of new knowledge. CCG is especially actual in analysis and interpretation of extreme situations in realtime IS. Reliable estimation of situation and acceptance of reasoned decisions in such systems substantially depend on effective solution of situation identification problem.

Problems of CCG are connected with solution of the following problems [7]:

- development of knowledge representation models ensuring uniform objects representation of algebraic and geometrical thinking;

- visualisation of knowledge, whose textual descriptions is complicate;

- search of transition ways from observable images to hypothesis about latent processes formulation.

In the paper different ways of problems solution and construction of cognitive images are considered by the example of functioning onboard intelligent systems of navigation safety and aircraft landing [6,7]. Thus three applications of cognitive structures are shown:

- visualisation of multivariate information at interpretation of dynamic measurements;

- representation of information for operative decisions acceptance in extreme situations;

- information compression for construction and training of artificial neural networks (ANN).

Applications of carried out researches are IS using parallel algorithms of measuring information processing on the basis of supercomputer technologies.

\section{General Approach}

CCG is a synthetic scientific field. It uses tools of functional analysis, both analytical and algebraic geometry and statistics from the one side, and discrete mathematics, theory of graphs and scientific visualisation from the other side. Identification and decoding of cognitive structures are carried out both on stage of knowledge system formalisation during IS synthesis, and on stage of testing by methods of imitating modelling.

Algorithm of cognitive model construction can be realised by sequence of procedures reflecting technology of knowledge representation about DO functioning $[7,8]$ :

$$
\begin{gathered}
(q, U) \underset{E}{\longrightarrow} J(q \in Q) ; \\
(J, U) \underset{S}{\longrightarrow} \Omega ;(\Omega, D) \longrightarrow{ }_{P}^{\longrightarrow} R ; \\
(R, U) \underset{A D}{\longrightarrow} W
\end{gathered}
$$


Here $\mathrm{q}$ is the subregion of a subject domain Q; $\mathrm{U}$ is the problem of DO control; $\mathrm{J}$ is the visualised image; $\mathrm{W}$ is the program; $\mathrm{E}$ is the heuristic procedure of mapping $\mathrm{q}$ in $\mathrm{J} ; \mathrm{S}$ is the procedure of programs synthesis; D is the data array which is the basis for solution search during modelling; $\mathrm{P}$ is the solution searching procedure; $\mathrm{R}$ is the result; $\mathrm{AD}$ is the procedure of solution acceptance; $\mathrm{W}$ is the cognitive structure.

The general scheme of cognitive model, is characterised by the system of functional equations:

$$
\Phi_{j}=t_{i}\left(F_{i}, f_{j}\right), i=1, \ldots, k, j=1, \ldots, n
$$

where $t_{i}$ are the terms constructed with the help of composition and recursion; $F_{i}$ are the functional variables of corresponding processes in system (1); $f_{j}$ are the functional constants corresponding to elementary processes.

Procedure $\mathrm{W}$ is most labour consuming and poorly known. Construction of $\mathrm{W}$ is connected with specificity of considered initial information in real-time onboard IS. Due to complexity of "exact" physical-mathematical model of DO-environmental interaction $\Phi_{\mathrm{j}}$ let us consider metaphorical representations of $\mathrm{W}$ on the basis of initial data analysis.

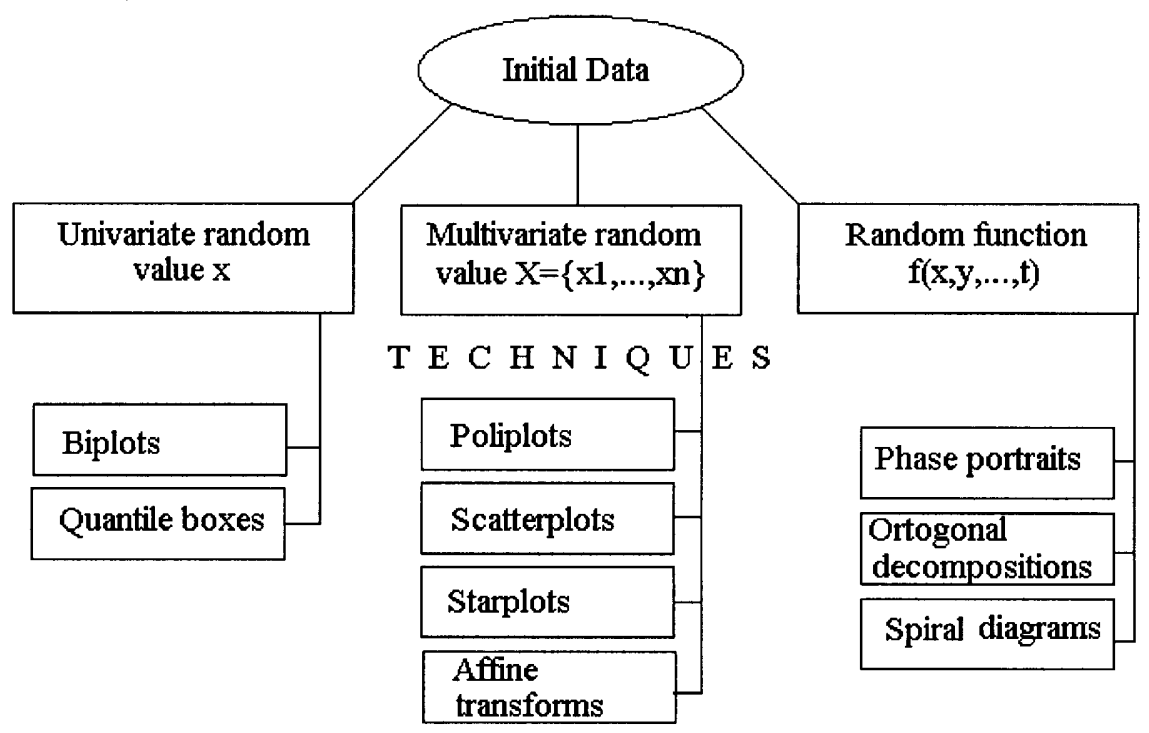

Fig. 1. Models of data and techniques of cognitive images generation

Probabilistic measure could be considered for majority of environmental processes. In this case power tools of multivariate statistics $[9,13]$ permit to construct procedure W. Multivariate statistics operates by canonical variables (principal components, factor loadings, canonical correlations), uses analyses of regression, variance and covariance, employs classification and discrimination tools. This methodology was already developed well in terms of random value (RV) and 
multivariate random value (MRV) models. However, application of such methods for random function (RF) or time series model (that characteristic for real time IS) demands updating of these procedures.

In this paper basic approaches to identification and construction of cognitive structures are presented in terms of RV, MRV and RF models. Structure of used models and corresponded techniques is shown in the fig. 1.

Techniques shown in fig. 1 are generally considered in paper [4]. One set of them (biplots, polyplots, affine transform, spiral diagram) is based on the idea of special functional transformation of initial data to easily recognised image (e.g., straight line). Second set contains all methods (quantile boxes, orthogonal decompositions, phase portraits) which are based on the elimination of low-informative variables. And the third set of methods (scatterplots, starplots) results in plane representation of nonplanal (multivariate) images.

From the other side such measure could be fuzzy for processes which connected with human actions (driver, pilot, navigator et al.).

\section{Applications}

Below several examples of CCG structures $\mathrm{W}$ in (1) are present. IS of ship seaworthiness (safety in a seaway) and IS of aircraft landing in sea conditions are considered. These examples demonstrate CCG-principles for visualisation of some informative probabilistic characteristics of both DO and environment for providing effective operator-computer interaction and support of control decisions.

\subsection{Cognitive structures for visual monitoring of DO-enviromental interaction in a sea}

Main external factors determining DO behaviour (e.g. ship) in operation are wind and sea waves. Let us consider features of interpretation of these characteristics with the help of CCG images. Initial information about variability of wind and wave excitations is formed on the basis of dynamic measurements. It is used in models of inference production rules. Results of imitating modelling of complex dynamic scenes of DO-environment interaction and various fragments of CCG images generated with the help of specially developed algorithms are represented on display together with numerical information.

Wind waves representation. Wind waves have complicated spatial structure. Geometrical characteristics of waves are determined by spatial spectral density $\mathrm{E}_{\zeta}(\mathrm{u}, \mathrm{v})$, or correlation surface:

$$
K_{\zeta}(r, \theta)=D_{\zeta} \exp \left(-\alpha(\theta) r^{1+\varepsilon}\right) \cos (\beta(\theta) r)
$$


where $r=\sqrt{x^{2}+y^{2}}, \theta=\arctan (y / x), D_{\zeta}$ is the waves variance, $\alpha(\theta)$ is the decrement of decay, $\beta(\theta)$ is the fluctuation frequency in direction $\theta, \varepsilon$ is a small constant.

Specific wave size in direction $\theta$ is:

$$
\lambda(\theta)=\frac{\pi}{\beta(\theta)}
$$

Here value $\lambda(0)$ is traditional definition of wavelength. So, cyclic spatial structure of wave field could be described by curve in polar co-ordinates, as geometrical place of points $\{\theta, \lambda(\theta)\}$. In simple case (for visualising purpose) let us consider such structure as rotated ellipse with semiaxes $\{\lambda(0), \lambda(\pi / 2)\}$.

One of the dangerous situations is ship movement along general direction of waves with speed closed to phase speed of waves. The length of dangerous wave is close to length of the ship. So, for visualising of such scenario, let us superpose the above mentioned ellipse of wave "hump" and stylistic ship image. Orthogonal axes of the plot mean module of ship speed $\mathrm{V}$ and cosine of course angle $\chi$ (in geographical coordinates), see fig. 2. From the fig 2(a) we can see that ship length is close to dimension of wave humps, and situation must be considered as dangerous.

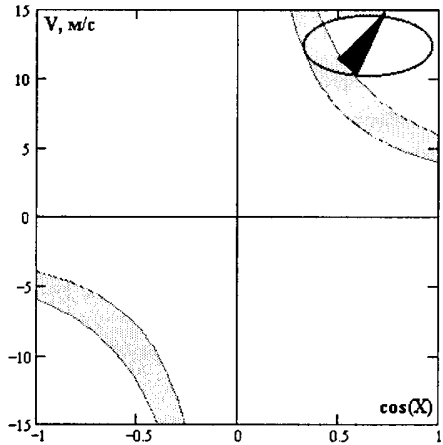

(a)

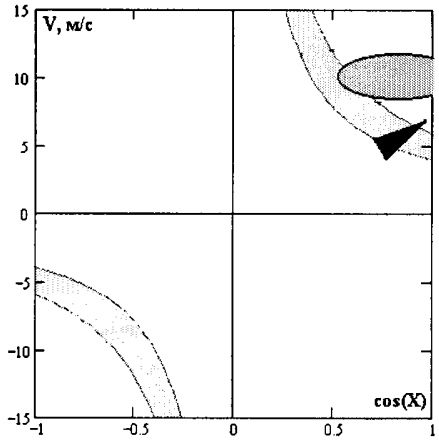

(b)

Fig. 2. Influence of environmental on ship.

(a) - danger of broaching (on quartering waves), (b) - danger of main resonance.

Resonant regimes of ship motion. Identification of ship motion resonant regimes is based on the condition (for rolling):

$$
\left|\omega-\frac{V}{g} \omega^{2} \cos \chi-k n_{\Theta}\right|<\varepsilon
$$

where $n_{\Theta}$ is the natural frequency of main rolling resonant, $k$ is the coefficient of overtones, $\mathrm{v}$ is the ship speed, $\varepsilon$ is a small constant for comparison. After transformation $\mathrm{u}=\mathrm{v} \cos \chi$ we obtain: 


$$
u v=\left(\omega-k n_{\Theta} \mp \varepsilon\right) g / \omega^{2}
$$

Equation (6) defines two hyperbolic domains where resonance condition (5) is valid. Value $\varepsilon$ of these domains approximately depends from sea state:

$$
\varepsilon=0.43 h_{s}^{-1 / 2}
$$

where $h_{s}$ is the significant wave height.

If in (6) we fix $\omega=\omega_{\mathrm{m}}\left(\omega_{\mathrm{m}}\right.$ is peak spectrum frequency), than two "dangerous" domains are observes on plane (u,v). After functional transformation we will have the same on plane $(\mathrm{V}, \cos (\mathrm{X}))$. Dangerous resonant situation in quartering directions is shown in the fig 2(b).

\subsection{Cognitive structures for landing of sea-based aircraft}

Landing of aircraft in sea conditions is one of the complex extreme situations connected with flights safety. Features of landing in complicate hydrometeorological conditions are not connected with ship navigation to the certain direction in accordance with wind direction only, but also with choice of optimum direction of aircraft (helicopter) flight in which variability of wind would be the least $\left(\mathrm{D}_{\min }\right)$. This direction usually essentially differs from direction of mean wind speed vector. Since component method of wind speed analysis does not permit to determine this value, let us use advantage of vector algebraic method for vector processes analysis.

Let $\vec{V}$ is planar vector of wind speed with parallel and meridian components $\mathrm{V}_{\mathrm{x}}$ and $\mathrm{V}_{\mathrm{y}}$. Then mathematical expectation

$$
\vec{m}_{\triangleright}=M\left(\begin{array}{l}
V_{x} \\
V_{y}
\end{array}\right)
$$

is planar vector too, and correlation function

$$
K_{\bar{v}}=\left(\begin{array}{cc}
K_{V_{x}} & K_{V_{x} V_{y}} \\
K_{V_{y} V_{x}} & K_{V_{y}}
\end{array}\right)
$$

is dyadic tensor with the components $\mathrm{K}_{\mathrm{vivj}}$. Variance $\mathrm{D}_{\overrightarrow{\mathrm{v}}}=\mathrm{K}_{\overrightarrow{\mathrm{v}}}(\bullet)$ when $\bullet=0$. Hence, variability of wind speed is defined by set of five values: module $\left|\vec{m}_{\mathcal{D}}\right|$ and direction $\bullet$ of mean wind speed, and invariants $\bullet_{1}, \bullet_{2}, \bullet$ of variance tensor:

$$
\begin{gathered}
\left|\vec{m}_{\nabla}\right|=\sqrt{m_{V_{x}}^{2}+m_{V_{y}}^{2}}, \\
\bullet=\arctan \frac{V_{x}}{V_{y}}
\end{gathered}
$$




$$
\begin{aligned}
& \bullet{ }_{1,2}=0.5\left(I_{1} \pm \sqrt{I_{1}^{2}-4 I_{2}}\right) \\
& \bullet=0.5 \arctan \left(\frac{2 D_{V_{x} V_{y}}}{D_{V_{y}}-D_{V_{x}}}\right)
\end{aligned}
$$

where $I_{1}, I_{2}$ are linear and quadratic invariants of tensor (9).

Tensor (9) is conveniently represented as ellipse with semiaxes $\lambda_{1}, \lambda_{2}$ for characterisation of $\vec{V}$ intensity in different directions,. Orientation of largest semiaxis is $\alpha \pm 180^{\circ}$. Example of wind field parameterisation in cyclone in the Mediterranean is shown in the fig. 3. Mean wind speed vectors are plotted as arrows, and standard deviations are plotted as ellipses.

Obviously that $\mathrm{D}_{\min }=\lambda_{2}$ and optimal landing direction is $\alpha \pm 90^{\circ}$. Thus, conventionalised graphic images of ship, mean wind speed vector, helicopter and standard deviation ellipse (fig. 4) are offered for operator (manager of landing) for acceptance of the decision of choice of optimum course angle and ship speed for helicopter landing.

Other way of CCG images using at aircraft landing is described in papers [7,14]. The approach is developed on the basis of the patent of Yu. Nechaev ensuring situation analysis and acceptance of the decisions during realisation of landing operations in sea conditions. The analysis is connected with recognition of wave field structure and of behaviour of flight deck in various external excitation conditions. Developed algorithm permits to recognise regimes of ship motion and "window of safe landing" during formation of command to the pilot about operation beginning and finalising. Display control is provided by the computer program, which carries out all necessary operations on information processing, situation estimation and generation of practical recommendations in real time.

\subsection{Cognitive structures for learning ANN identifying extreme situations}

In onboard IS CCG structures are not used only for analysis and interpretation of extreme situations. It is possible use it in processing of measuring information for representation of input signals for ANN. Effect of CCG application consists of "compression" of information, simplification of ANN configuration and reduction of learning time. Such approach is very useful for the problem of damaged ship state identification (flooding).

Solution of recognition problem by means of CCG was carried out with the help of transformation of information in terms of formally logic description. Search of concrete model of image was carried out with the help of cognitive spiral permiting "to compress" initial information about nonlinear ship rolling with stochastic variability of periods. Application of cognitive spiral to stochastic processes resulted in "alignment" of periods and their affine transformation to one value. 


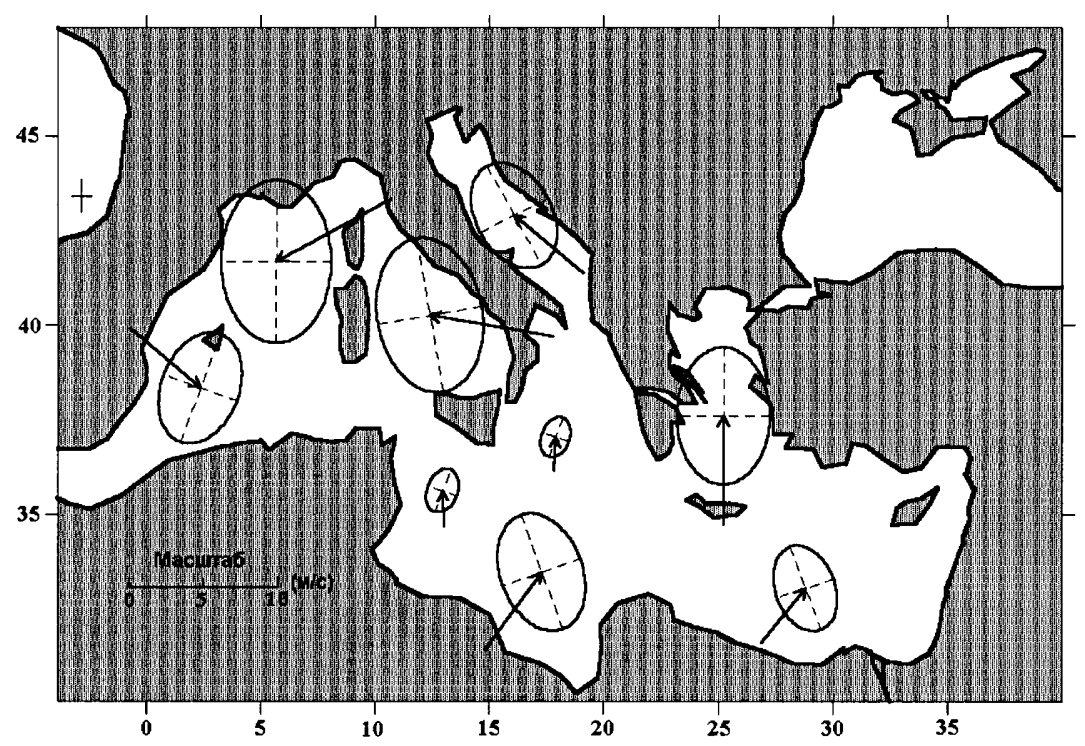

Fig. 3. Cognitive images of wind speed field. Cyclone in the Mediterranean, 1.12.1969

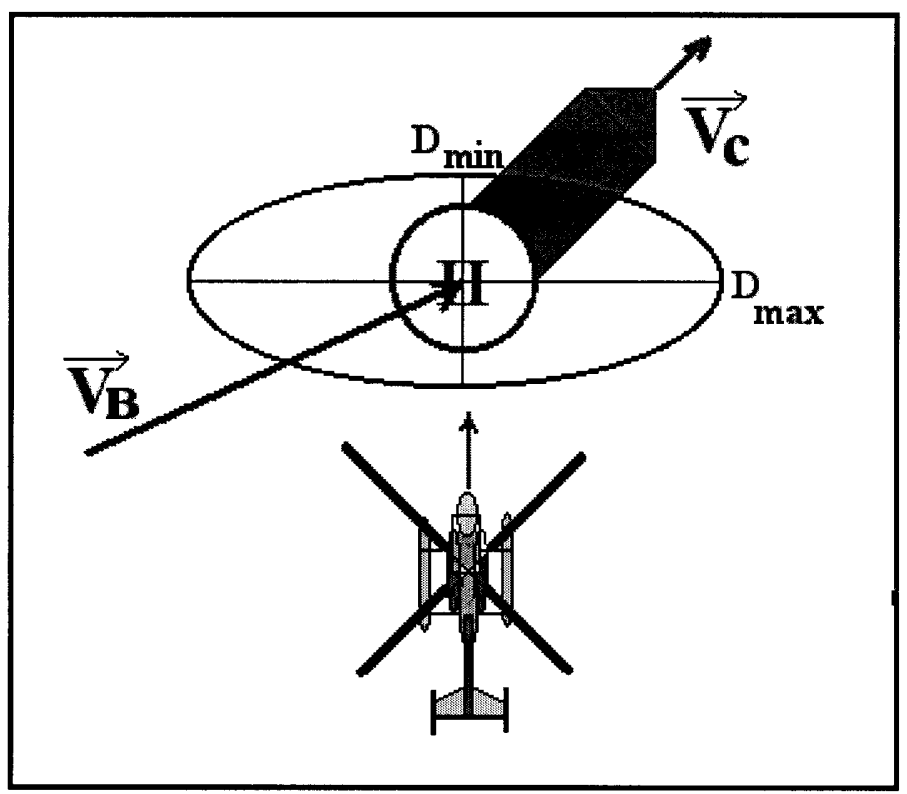

Fig. 4. Cognitive image for ship navigation to direction of wind speed $\vec{V}_{B}$, with account of minimal atmospheric turbulence.

Cognitive spiral, as alternative to a phase portrait, contains more information, which it is possible to select visually orienting on image structure. Breadth of bands 
in spiral, its saturation by colour and frequency of changing of band of one colour by band of other colour can be referred to such information. Asymmetry of upper and lower parts of cognitive spiral and distribution of colour on angles close to 0 and 180 degrees can also play important role.

Considered problem of recognition is connected with classification of typical cases of flooding. There are five classical situations of damaged ship. The first case is trivial enough: lack of static heel at symmetric flooding and positive initial metacentric height. In other emergency states ship always has heel on a side. It allows to easily pick out first case without using complicated inference procedures. As regards to the fifth case it is usually considered as subset of fourth case. Similar pictures of oscillation are typical for this case.

Analysis of CCG structures permits to obtain information for recognition. Rational decision of this problem is obtained by consideration of characteristic sections in cognitive spiral at various corners. As a result we did not exploit basic characteristics of CCG (human-computer interaction), but we used above mentioned CCG images for ANN learning. Such information permits to identify constant number of entrance neurons, to simplify structure of ANN and to have freedom in choice of length of initial time series. Cognitive image of ANN learning on the basis of two-layer map of Kohonen is shown in the fig. 5.

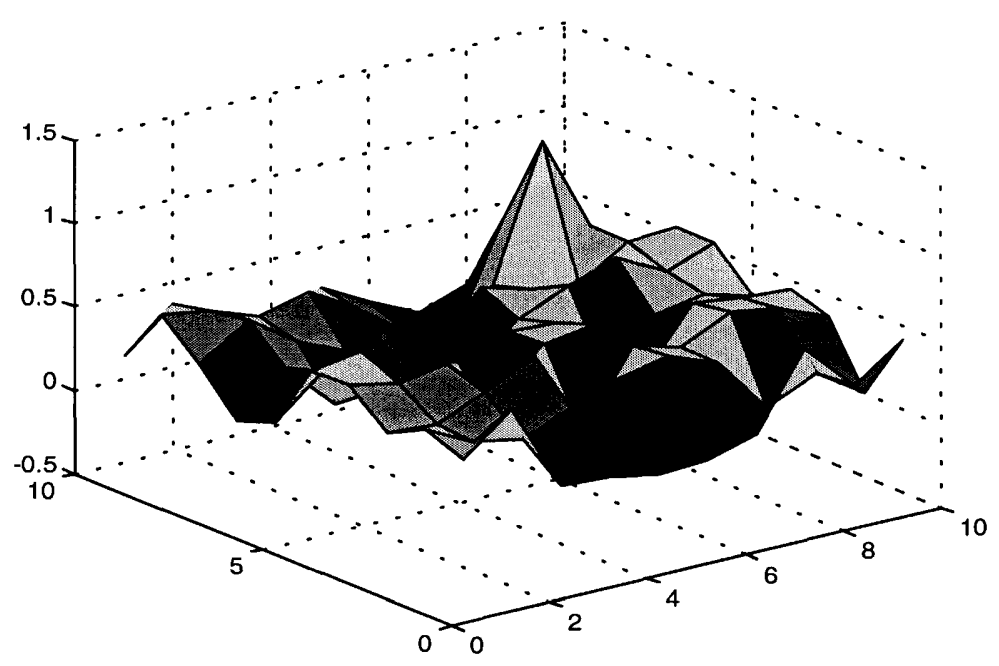

Fig. 5. Cognitive image of ANN training. Two-layer map of Kohonen (10x10 neurons)

\section{Conclusions}

Carried out investigation testifies to the large opportunities of CCG methods for problems of data interpretation in onboard real time IS. Methods of CCG improve 
efficiency of recognition and analysis of latent structures describing DO- environment interaction. Especially important role of CCG images application is for analysis and forecast of extreme situations connected with safety of ship navigation and aircraft landing in sea conditions. "Compressed" and easily interpretive cognitive representations of dynamics stimulate image thinking and allow to operate with representational objects of abstract nature.

The research is supported by grants of RFBR N 00-07-90227 and INTAS 1999 N666.

\section{References}

1. Albu V.A., Choroshevsky V.F. COG-system of cognitive graphics. Developing, realization, application. Izv. AS USSR, Technical cybernetics, 1990, 5, 37-43.

2. Bashlykov A.A., Pavlova E.B. Intellectual user interface of system SPRINT-RV on the base of cognitive graphics. Proc. of V Int. Conf. CAI96, Kazan, Russia, 1996, 3, 385-386.

3. Boult T.E., Fenster S.D.and Kim J.W. Dynamic attributes, code generation and the IUE, DARPA 94. vol.1,p.p.405-422.

4. Chamber J.M., Cleveland W.S., Kleiner B, Tukey P.A. Graphical methods for data analysis. Belmont, CA: Wadsworth, 1983.

5. Cognitive science. An introduction a bradford book // The MIT Press CambridgeMessachusetts-London-England.1989.

6. DegtyarevA.B., Dmitriev S.A., Nechaev Yu.I. Methods of cognitive computer graphics for analysis of dynamical object behavior. Proc of II Int. Conf MORINTECH-97, St.Petersburg, Russia, 1997, 6, 83-87

7. Intelligence system in marine research and technology /Ed. by $\mathrm{Yu}$. Nechaev. St.Petersburg. SMTU. 2001 (in Russian).

8. Ivanischev V.V. Objects modelling on the visualisation. Proc. of II artificial intelligence conference. Minsk.1990, pp.122-124.

9. Johnson R.A., Wichern D.W. Applied multivariate statistical analysis. Prentice-Hall International, Inc., London, 1992, 642 pp.

10. Lakoff J. Cognitive Modelling. Proceedings of language and intelligence.-Moskow. Progress.1996.

11. Lowton D.T., Dai D., Frogge M.A., Gardner W,R., Pritchett H., Rathkopf A.T. and TenG. The IUE User Interface, DARPA 93, p.p.289-300.

12. Magasov S.S. Functional sceme of cognitive model. Artificial Intelligence. 3, 2000, pp.257-262.

13. Mardia K.V. Kent J.T., Bibby J.M. Multivariate analysis. London, Academia Press Inc., 1979

14. Nechaev Yu., Degtyarev A., Boukhanovsky A. Coignitive computer graphic for interpretatrion of information on real time intelligence system. Proc. of III Int. Conf. MORINTEX-99. St.Petersburg. 2001. Vol.1, pp.297-303.

15. Shneiderman B. Perceptual and cognitive issues in the syntacsic. Semantic model of programmer behaviour. Proc. of symp. on human factors and computer science. Human Factor Society. Santa Monica, California,1978.

16. Zenkin A.A. Cognitive computer graphics. M., Science, 1991 (in Russian)

17. Zenkin A.A. Cognitive computer graphics - application to decision support systems. Proc of II Int. Conf MORINTECH-97, St.Petersburg, Russia, 1997, 8, pp.197-203 